\title{
Neoadjuvant treatment is always justified for small PDAC, especially for clinical T1? Debate from the position of Cons
}

\author{
Hiroki YAMAUE*
}

Second Department of Surgery, Wakayama Medical University, Japan

Lecture: What's the appropriate treatment for resectable pancreatic cancer? The patients have to have R0 operation, and we have been using the mesenteric approach to enhance the incidence of R0 operation. What about the clinical impact of neoadjuvant chemotherapy and chemoradiotherapy, especially for small PDAC, T1 cancer. In this debate session, I will discuss the justification of neoadjuvant treatment as a position of Cons.

Even in the patients with resectable pancreatic cancer, especially considering distal pancreatectomy for body and tail pancreatic cancer with radiographic splenic vein or artery involvement, the survival of patients with radiographic splenic vein or artery involvement are almost similar to survival of BR cancer. The MST was 23.4 months and 15.1 months, respectively. Therefore, radiographic splenic vein or artery involvement is definitely poor prognostic factor, and neoadjuvant treatment should be mandatory for longer survival even in resectable cancer with body and tail pancreatic cancer if the tumor invade the splenic vein or splenic artery radiographically (Kawai, Yamaue et al. Ann Surg Oncol 2020).

The topic of randomized controlled trial for resectable cancer is reported from the Dutch PREOPANC trial of neoadjuvant treatment for patients with both resectable cancer and borderline resectable cancer (PREOPANC trial, J Clin Oncol 2020). In both overall survival and disease-free survival, the patients with neoadjuvant treatment had longer survival compared with immediate surgery. However, this advantage of neoadjuvant treatment indicates in the mixed groups of resectable and borderline resectable cancer. The subgroup analysis in resectable pancreatic cancer, MST in preoperative treatment group was 14.6 months and in immediate surgery group was 15.6 months. Also, disease-free survival was 9.2 months and 9.3 months, respectively. Moreover, the incidence of R0 operation was also quietly similar in both groups, $66 \%$ and $59 \%$, respectively. This data clearly shows that there is no survival-impact of neoadjuvant treatment in resectable pancreatic cancer. The Japanese study of Prep-02/JSAP-05 was a randomized controlled trial with comparison of upfront surgery, that means no neoadjuvant chemotherapy, and NAC-GS, with neoadjuvant chemotherapy using gemcitabine plus S-1. The overall survival was longer in NAC group compared with upfront surgery group, but these groups partially contain the borderline resectable-portal vein (BR-PV) patients. In the patients with the tumor size less than $2 \mathrm{~cm}$ and T1 tumor had no statistical difference between up-front surgery group and NAC group because the $95 \%$ confidence interval cross the line of 1.0 . Thus, the clinical impact of neoadjuvant treatment might be very limited in patients with resectable pancreatic cancer, especially small cancer less than $2 \mathrm{~cm}, \mathrm{~T} 1$.

The conclusion of the debate from the position of Cons is; neoadjuvant treatment might not be mandatory in T1 resectable pancreatic cancer. Who should have a clinical benefit by neoadjuvant treatment with resectable cancer? We have to learn and consider the molecular diagnosis including liquid biopsy to measure circulating tumor DNA. 